\title{
Multislice computed tomography imaging of gastrointestinal stromal tumors
}

\author{
Nadir Sezer ${ }^{1}$, Muhammed Akif Deniz ${ }^{2^{*}}$, Zelal Taş Deniz ${ }^{2}$, Cemil Goya ${ }^{3}$, Eşref Araç ${ }^{4}$, Mehmet Emin $^{2}$ \\ $\operatorname{Adin}^{5}$ \\ ${ }^{1}$ Department of Radiology, Van Education Research Hospital, Van, Turkey \\ ${ }^{2}$ Department of Radiology, Health Scuence University Gaz̧i Yaşargil Education Research Hospital, Diyarbakur, Turkey \\ ${ }^{3}$ Department of Radiology, Dicle University School of Medical Science, Diyarbakir, Turkey \\ ${ }^{4}$ Department of Internal Medicine, Health Scuence University Gazi Yaşargil Education Research Hospital, Diyarbakur, \\ Turkey \\ ${ }^{5}$ Department of Radiology, Silvan Dr. Yusuf Azizoğlu State Hospital, Diyarbakur, Turkey
}

\begin{abstract}
Gastrointestinal stromal tumors (GIST) are mesenchymal tumors that constitute 1-3\% of primary gastrointestinal tumors and approximately $5 \%$ of all sarcomas of the gastrointestinal tract. Multislice Computed Tomography (MSCT) is a highly sensitive imaging technique for detecting GISTs.

We retrospectively evaluated findings of 56 consecutive subjects that were examined at Dicle University, School of Medicine, between 2008-2015 and diagnosed with GIST. Lesions were divided into two clinically distinct entities as recurrent and primary lesions. Densities of lesions in comparison to liver, origins of and spreading of the lesions, dimensions of the lesions, behavior pattern, and factors such as contour properties, invasion, and calcification which could potentially indicate malign behavior were also evaluated.

Age span of included subjects were between 28 and 81 . The most common tumor location for hollow organs was found to be stomach $(n=19,32 \%)$. In extra-luminal, regions the most common tumor location was found to be mesentery $(n=4)$. Hansfield Unit densities of tumoral lesions in comparison to liver density were hypodens ( $n=32,71 \%)$, isodens ( $n=9,20 \%)$ and hyperdens $(n=4, \% 9)$ in a descending order. The tumor invasion was found to be effecting liver, peritoneum, and bladder, in a descending order. In $11(24 \%)$ subjects, distant metastasis to the liver was evident. In $4(9 \%)$ subjects peritoneum, in $3(7 \%)$ subjects adrenal gland and in $1(2 \%)$ subject bone metastasis was evident.

MSCT is an important and indispensable modality for the detection, localization and identification of GISTs that can be seen in different parts of the gastrointestinal tract.
\end{abstract}

Key Words: Multislice computed tomography, gastrointestinal stromal tumor, viseral sarcoma, gastrointestinal system

\section{Introduction}

Gastrointestinal stromal tumors (GIST) are mesenchymal tumors that constitute $1-3 \%$ of primary gastrointestinal tumors and approximately $5 \%$ of all sarcomas of the gastrointestinal tract (1). The current data indicate a prevalence of 20$40 / 1,000,000$ (2). In the United States, 1520/1,000,000 new cases are reported annually (1). It is seen at approximately the same rate in males and females $(1,3)$. The diagnosis age is usually over 50 , and the maximum incidence is in $5^{\text {th }}$ and $6^{\text {th }}$ decades. Approximately $20 \%$ of cases are under the age of 40 , and the number of cases under the age of 20 is quite few $(1,3)$.

In more than $80 \%$ of cases, the lesions are located in gastrointestinal tract (2). The tumor may originate from anywhere in the gastrointestinal tract, from the esophagus to the anus (1). The most frequent locations are stomach (40-70\%), small intestine (often jejunum) (20-50\%), colonrectum (5\%), and esophagus (less than 5\%). GISTs are observed in the retroperitoneal area and minor pelvis at a rate of $10 \%$.

Multislice Computed Tomography (MSCT) is a highly sensitive imaging technique for detecting GISTs and could detect an abnormality in about $87 \%$ of cases $(4,5)$. On CT, GISTs could be seen as intramural masses showing uniform sharp homogeneous attenuation; or as heterogeneous masses of various thicknesses and contrast enhancement, irregular central fluid densities due to tumor necrosis, and oral contrast agent filling in tumor due to necrotic tumor surface or rarely 
air. Computed tomography (CT) has high sensitivity in detecting metastatic lesions (6-10).

The aim of this study was to describe radiological findings of GIST lesions using MSCT and to correlate these findings with demographic data and pathologic mitotic indices.

\section{Materials and methods}

A total of 45 patients who had been diagnosed with GIST between 2008 and 2015 were included. Contrast-enhanced abdominal CT images of patients, obtained using 16-detector (Toshiba Activion V3.00, 2010) and 64-detector (multislice Philips Brillance V2.6.1 2007) CT devices, were retrospectively reviewed.

Subjects were scanned from xiphoid process of sternum to the end of the symphysis pubis. The images were obtained with 16- and 64-detector CT devices using the following parameters: $\mathrm{FOV}=350-420 \mathrm{~mm}$, voltage $=120 \mathrm{kv}, \mathrm{mAs}=250$ mas/slice, collimation $=64 \times 0.625$ and $16 \times 1.0 \mathrm{~mm}$, pitch value $=1$, rotation time $0.75 \mathrm{sec}$ (16-detector ct)-1 sec (64-detector ct), section thickness $=0.9 \mathrm{~mm}$, window width $/$ level $=360 / 60$. The cases were injected with 1-1.5 cc IV contrast agent per kilogram $(\mathrm{kg})$ manually at a rate of 2$2.5 \mathrm{ml} / \mathrm{sec}$. After contrast agent injection, the slices were obtained with a delay of $40-65 \mathrm{sec}$. The abdominal CT images of patients in our study group were evaluated either in work station (Philips Extended Brilliance Workspace Philips Medical Systems, Best The Netherlands) or using online archive system (PACS, infinit INFINITT Healthcare Co., South Korea).

Our study protocol was approved by the local ethics committee as a retrospective observational archival study.

The patients included in this study were evaluated as two separate clinical entities: primary and recurrent disease. Those who had been evaluated using MSCT at the time of initial diagnosis and whose preoperative radiological examinations existed were considered as primary. Those who had been previously imaged or operated on in another medical center and had presented to our hospital with recurrent tumor were considered as recurrent tumor.

Density of tumoral mass compared to liver was measured in Hansfield units in post-contrast series. The masses with qualitatively higher densities compared to liver parenchyma were considered as hyperdense tumoral masses, those with equal densities were considered as isodense, and those with lower densities were considered as hypodense.

The gastrointestinal organ or segment in which the main part of the tumoral mass was located was accepted as the origin of the mass. The mass extension was assessed according to the wall of the luminal organ in which the mass was located in. Thus, the masses that developed within the gastrointestinal segment wall and caused only expansion of the wall, were considered as "limited to wall" masses. Those extending through adjacent anatomical structures were considered as "exophytically extended" masses, and those extending into the lumen were considered as "endoluminally extended" masses. Furthermore, lesions located outside the lumenal organs were considered as extraluminal.

The two greatest dimensions of the mass were calculated in millimeters in axial, coronal, or sagittal planes. Behavior of the mass and the findings that may indicate malignancy were studied in the following way: the region with significantly lower density compared to the main mass or with density of liquid was accepted as radiological necrosis; the contour properties of the mass were assessed quantitatively as regular or irregular; the progression of the mass through adjacent anatomical structures without interrupting the connection of the main tumoral mass was considered as invasion; formations within the mass with significantly higher density than blood density and bright enough to be quantitatively close to bone density, were considered as calcification.

Tumoral mitotic indices of the cases included in this study were recorded. The mitotic index was defined as the proportion of cells in metaphase (cells undergoing mitotic division out of all examined cells) to total cell count in percentage. The mitotic index classification was evaluated in three groups-low, medium, and high. (Those less than $5 / 50$ were low, those between $5 / 50$ and $10 / 50$ were considered medium, and those with a high rate of $10 / 50$ were considered high mitotic index).

Since this study is a retrospective observational study, incidence and percentage analysis of the data were primarily conducted. The statistical significance level was set as $5 \%$ (significant $\mathrm{p}$ value $<0.05$ ). Linear regression analysis was performed in order to evaluate the relationship of mitotic index with age, tumor size and density. Pearson's Chi-squared test was used to investigate the relationship between tumor size and gender. For all statistical operations and graphical 
visualizations, ' $\mathrm{R}$ statistical environment ( $\mathrm{R}$ studio for Mac OS Version 0.98.501C), 2009-2013)' or 'Microsoft Excel 2011 for Mac OS (Microsoft Corp., Redmond, Washington, ABD)' was used.

\section{Results}

The patients were in the age range 28 - 81 years (mean age: 60, standard deviation: 13.9). There were 28 males $(62 \%)$, and 17 females (38\%) females. Forty-one cases (91\%) were in the primary group and $4(9 \%)$ were in the recurrent group.

The most frequent location was stomach $(n=19$, $32 \%)$. Tumor was located in corpus $(n=12)$, antrum $(n=5)$, and rarely fundus $(n=2)$ regions of stomach. The least frequent location was colon. Moreover, in the colon, the rarest locations were the rectum and ascending colon. Tumors rarely originated from outside the luminal organs. In the extraluminal regions, the tumor was located most frequently in the mesentery $(n=4)$ and least frequently in the peritoneum $(n=1)$ and retroperitoneum $(n=1)$. The details of the tumor origins are summarized in Table 1.

Comparing densities of tumoral masses to the liver in post-contrast series in terms of Hansfield units, the relative densities were hypodense $(n=32$, $71 \%)$, isodense $(n=9,20 \%)$, (Figure 1) and hyperdense $(n=4,9 \%)$ (Figure 2 ). There was no statistically significant relationship between tumor density and mitotic index.

In terms of tumor sizes, lesions were observed to vary between 1.4 and $20.0 \mathrm{~cm}$. In statistical analysis, dimensional increase in tumor was found to have positive correlation with an increase in mitotic index $(p=0.002)$. There was no statistically significant relationship between tumor size and demographic variables such as age and gender ( $p=0.34,0.53$, respectively).

In terms of morphological findings that can indicate the nature of the mass, contour irregularity was detected in 17 cases (38\%). The contour irregularity was more frequent in cases with larger tumor size. Considering the largest axial dimension to be $5 \mathrm{~cm}$, there was a contour irregularity in only one of the cases below this limit $(n=14,7 \%)$, but in eleven of the cases above this limit $(n=31,35 \%)$. The dimensions of GIST lesions originating from outside of luminal gastrointestinal structures, called extraluminal in this study, were quite large compared to other lesions. The sizes of all extraluminal lesions were over $12 \mathrm{~cm}$, except for a single retroperitoneal case, which was about $6 \mathrm{~cm}$.

There was a low-density area consistent with necrosis in 12 cases $(27 \%)$. The main tumor was found to be a fistula with adjacent anatomical structures in 4 cases $(9 \%)$. The hyperdense focus consistent with calcification within tumoral mass was found only in 1 case $(2 \%)$.

Because of the disproportionate number of cases between groups, no statistical calculation could be conducted between recurrent and primary tumors (recurrent group $\mathrm{n}=4$, primary group $\mathrm{n}=41$ ). However, $100 \%$ of the recurrent tumors invaded adjacent organs, but $19 \%$ of the primary tumors invaded adjacent anatomical structures. In

Table 1. Lesions' origins and their incidences

\begin{tabular}{lcc}
\hline Origin & Number of cases (n) & Percentage (\%) \\
\hline Stomach (corpus) & 12 & 26.7 \\
Stomach (antrum) & 5 & 11.1 \\
Small intestine (jejunum) & 5 & 11.1 \\
Small intestine (ileum) & 5 & 11.1 \\
Mesentery & 4 & 8.9 \\
Duodenum & 3 & 6.7 \\
Stomach (fundus) & 2 & 4.4 \\
Colon (descending) & 2 & 4.4 \\
Colon (sigmoid) & 2 & 4.4 \\
Colon (rectum) & 1 & 2.2 \\
Colon (ascending) & 1 & 2.2 \\
Esophagus & 1 & 2.2 \\
Peritoneum & 1 & 2.2 \\
Retroperitoneal & 1 & 2.2 \\
\hline
\end{tabular}


descending order of frequency, invasive spread occurred through liver, peritoneum, and bladder. Lesions metastasized distantly to the liver in 11 cases $(24 \%)$. There was peritoneal metastasis in 4 cases $(9 \%)$, adrenal metastasis in 3 cases $(7 \%)$, and bone metastasis in 1 case $(2 \%)$.

\section{Discussion}

GIST, a visceral sarcomatous tumor, is rarely seen but it is the most common mesenchymal tumor of the gastrointestinal tract (11). The reported mean age of gastrointestinal stromal tumor onset is slightly above the mean age of our study. The location of the tumor is stomach $(60-70 \%)$, small intestine (20-30\%), colon (5\%), rectum and esophagus $(5 \%)$ in descending frequency (12). The most frequent location was also found to be

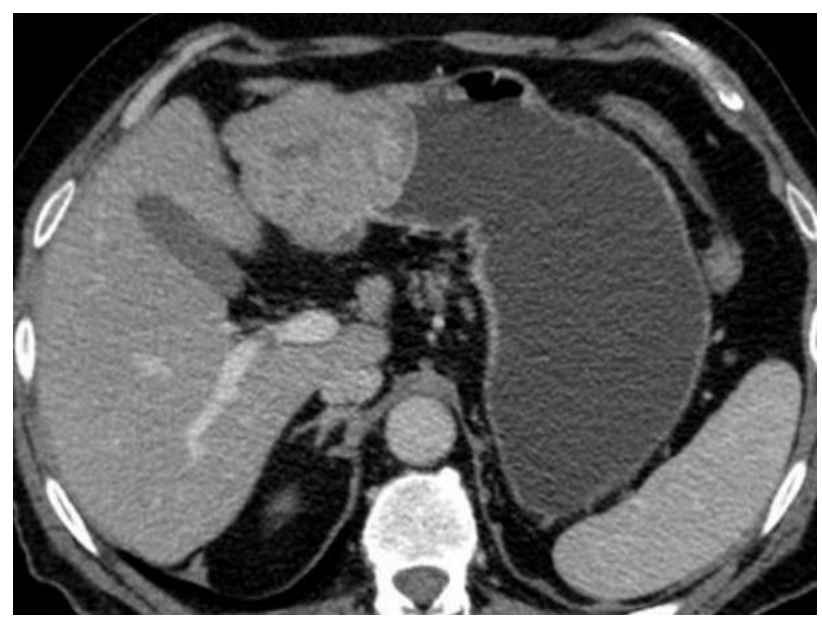

Fig. 1. 61-year-old male patient, mass which is isodense with liver and which extends into stomach antrum lumen.

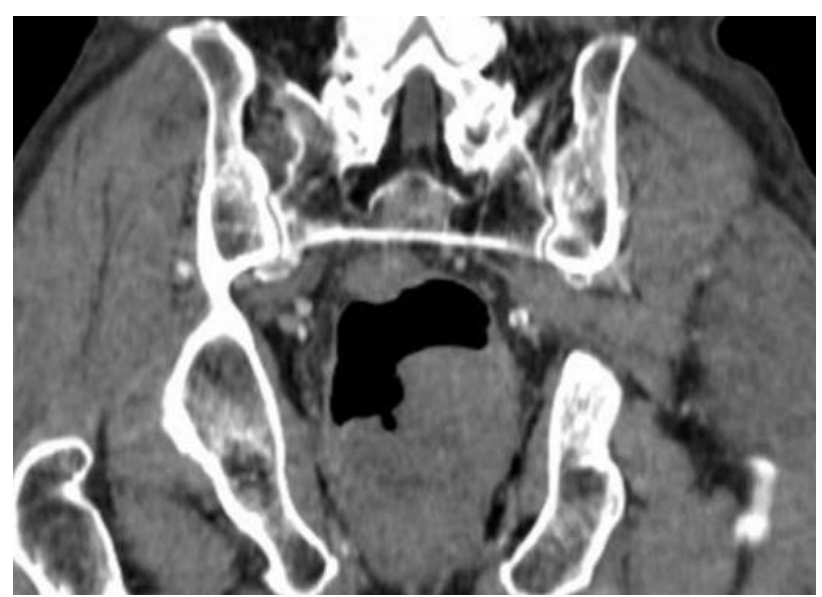

Fig. 3. 72-year-old male patient, endoluminal mass with rectal location. stomach in our study. Tumor was located in corpus, antrum, and rarely fundus regions of stomach in descending frequency. The least frequent location was colon. The rarest locations were rectum and ascending colon as segmental location (Figure 3). In the extraluminal regions, the tumor was located most frequently in the mesentery and least frequently in the peritoneum and retroperitoneum (Figure 4).

In our study, $91 \%$ of cases were in the primary group and $9 \%(n=4)$ were in the recurrent group. Considering the largest axial dimension to be 5 $\mathrm{cm}$, there was a contour irregularity in only one of the cases below this limit $(7 \%)$, but in eleven of the cases above this limit $(35 \%)$. The large- and small-sized tumors gave quite extensive CT findings in terms of tumor extension, lesion density, and aggressive findings that may indicate

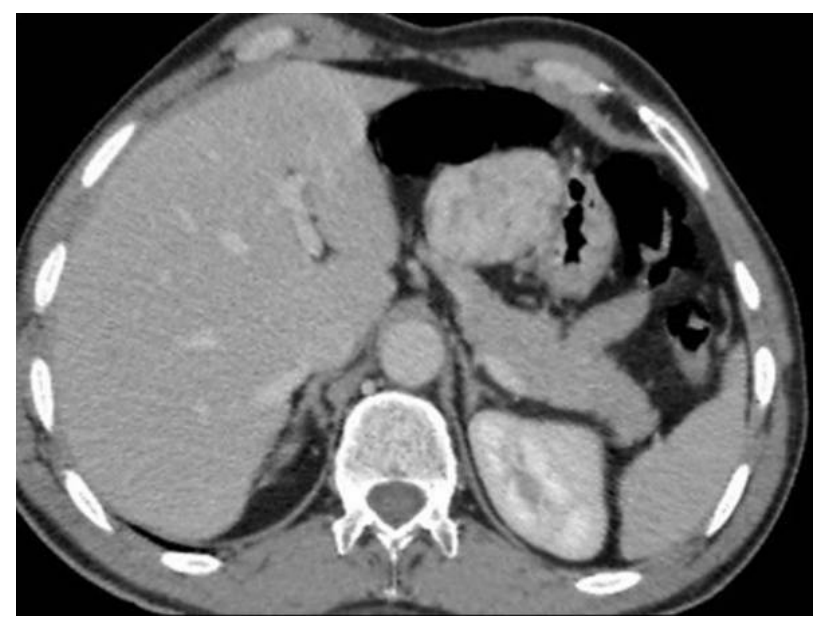

Fig. 2. 45-year-old female patient, mass which extends exophytically in stomach antrum and that is hyperdense compared to liver parenchyma.

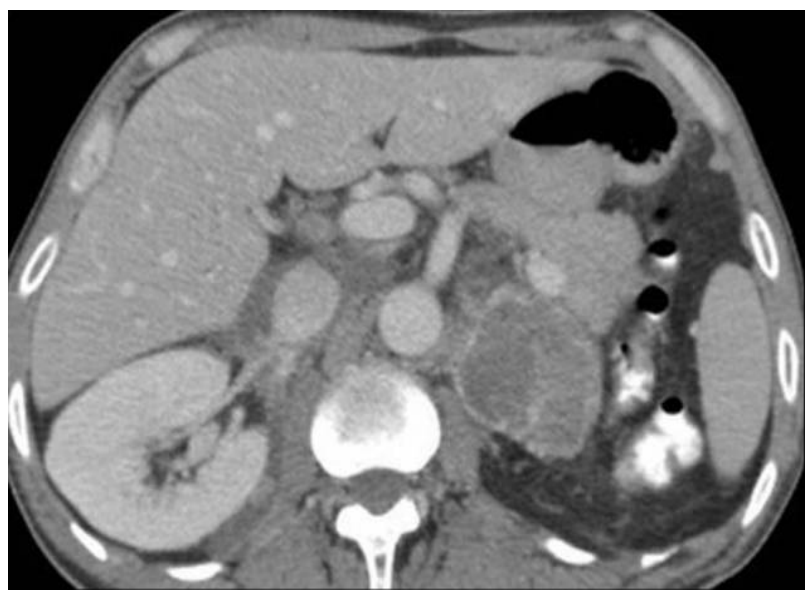

Fig. 4. 35-year-old male patient, necrotic mass in left retroperitoneal fatty tissue. 


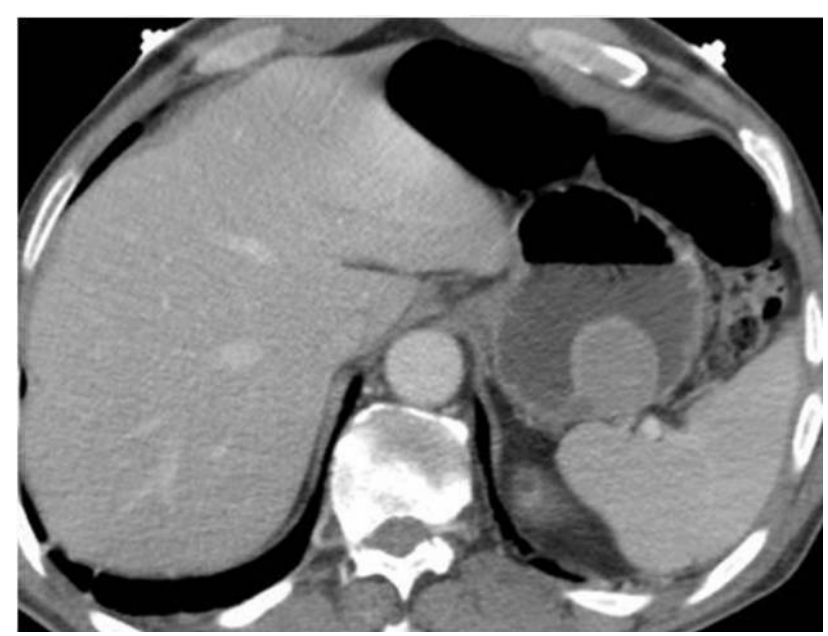

Fig. 5. 66-year-old male patient, hypodense mass with smooth contour in stomach corpus.

malignancy. In this study, there was a contour irregularity in only one case among small lesions, and this lesion was among the recurrent lesions. Comparing recurrent and primary tumors despite a significant difference in the number of samples in each group in this study, contour structure that may indicate malignancy, necrosis, and invasion to adjacent anatomical structures was more common in the recurrent tumor group. These findings were also significantly more frequent as tumor size increased regardless of whether the tumor was primary or recurrent. CT has been shown to be a very effective diagnostic test in differentiating primary and recurrent tumors taking advantage of the features described above.

We have found in this study that small-sized GIST (Figure 5) lesions $(<5 \mathrm{~cm})$ are more wellcircumscribed, the inner structure of the lesion is more homogenous, and they more often extend exophytically. There have been various results in various studies on exophytic or endophytic extension of small lesions in the literature. The small GIST lesions have been shown to have greater tendency to endoluminal extension in a study (13). An interesting finding of our study was that the dimensions of GIST lesions originating from outside of luminal gastrointestinal structures, called extraluminal in this study, were quite large compared to other lesions (Figure 6). The sizes of all extraluminal lesions were over $12 \mathrm{~cm}$, except for a retroperitoneal case. This finding may depend on the fact that the time to reach functional gastrointestinal symptoms for a lesion originating from the mesentery or peritoneum is longer compared to that of a lesion originating from the lumen itself.

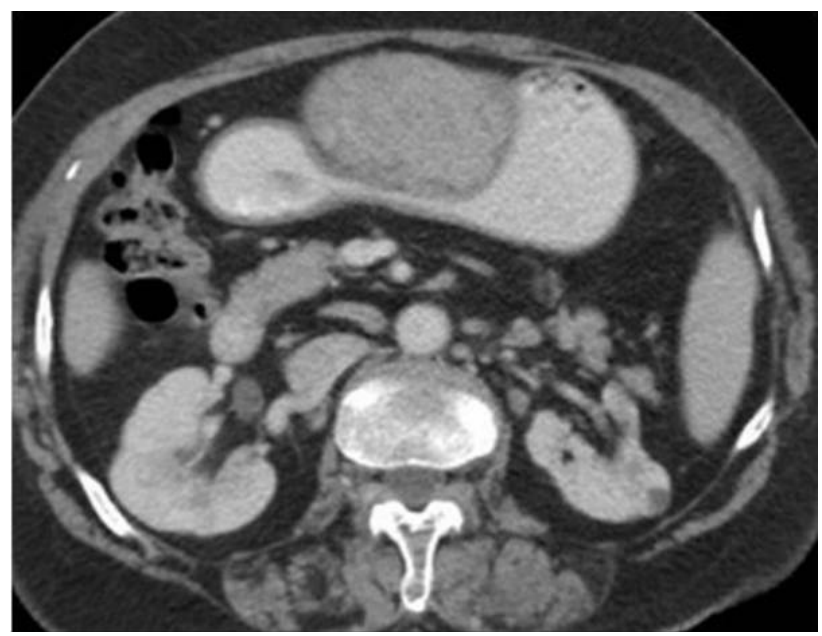

Fig. 6. 61-year-old female patient, exophytically extended smooth contour mass from stomach corpus.

The hyperdense focus consistent with calcification within the tumoral mass was found only in 1 case $(2 \%)$ in this study. There have been various results with this regard in the literature. While there was calcification in about $23 \%$ of small GIST lesions in one study, there was no calcification in any of the cases in another study (13-15). In a study including 19 cases, there was calcification only in one leiomyoma case (16).

In a study including 15 cases (17), there was fistula in lesion, and air fluid levels and air bubbles indicating deep ulceration in 2 of 15 cases. These air bubbles can also be seen in cases of superinfection within tumoral necrosis. In our study, there was a low-density area consistent with necrosis in 12 cases $(27 \%)$. The main tumor was found to be fistula with adjacent anatomical structures in 4 cases $(9 \%)$. Our findings are consistent with the literature. However one might bear in mind that the authors have indicated in their studies that these findings are not specific for GIST and could also be seen in lymphoma, metastatic disease, and adenocarcinoma of small intestine.

In this study, we also evaluated CT density of the tumor compared to liver density. That is, lesions that showed change in density in comparison to liver, besides necrotic areas, in post-contrast images may indicate a difference associated with the histological structure of the lesions. We compared this data with the mitotic index, the only histologic variable in our study. There was no statistically significant relationship between tumor density and mitotic index. Comparing densities of tumoral masses compared to liver in post-contrast series in terms of Hansfield units, relative 
densities were hypodense $(\% 71)$, isodense $(\% 20)$, and hyperdense (\%9) in descending frequency.

$\mathrm{CT}$ is a very effective method in detecting distant metastases and peritoneal spreads of leiomyosarcomas due to digestive system and genitourinary system $(4,5)$. In a study with 118 cases (18), there was liver metastasis in 29 cases $(25 \%)$ and peritoneal spread in 43 cases $(36 \%)$. Consistent with the literature, there was distant liver metastasis in 11 cases $(24 \%)$ in our study. The peritoneal metastasis was detected at a relatively low rate in our study, observed in 4 cases $(9 \%)$. There was an invasion of adjacent organs in 12 cases (27\% of lesions). While 100\% of recurrent tumors invaded adjacent organs, only $19 \%$ of primary tumors invaded adjacent anatomical structures. Invasive spread occurred in the liver, peritoneum, and bladder, in descending order.

The most important limitation of this study was its retrospective design. For this reason, there could be no long-term follow-up and thus no evaluation in terms of prediction of recurrence or remission risks. Since the examination of metastases was conducted only using CT findings, lesions that were too small to be detected with CT or whose density could not be distinguished from surrounding tissue may have not been detected in this study.

In conclusion, we have shown in this study that small-sized GIST lesions are localized, wellcircumscribed, and have relatively homogeneous structure. As lesion reaches larger sizes, it becomes subjected to internal changes such as necrosis, fistulization, invasion, and calcification. Furthermore, an increase in lesion size also brings malignant behaviors such as contour irregularity, and these can be detected with high precision using CT.

\section{References}

1. Duffaud F, Blay JY. Gastrointestinal stromal tumors: biology and treatment. Oncology 2003; 65: 187-197.

2. Cichoz-Lach H, Kasztelan-Szczerbinska B, Slomka M. Gastrointestinal stromal tumors: epidemiology, clinical picture, diagnosis, prognosis and treatment. Pol Arch Med Wewn, 2008; 118: 216-221.

3. Connolly EM, Gaffney E, Reynolds JV. Gastrointestinal stromal tumours. Br J Surg 2003; 90: 1178-1186.
4. Pidhorecky I, Cheney RT, Kraybill WG, Gibbs JF. Gastrointestinal stromal tumors: current diagnosis, biologic behavior, and management. Ann Surg Oncol 2000; 7: 705-712.

5. Jiang ZX, Zhang SJ, Peng WJ, Yu BH. Rectal gastrointestinal stromal tumors: imaging features with clinical and pathological correlation. World J Gastroenterol 2013; 19: 3108-3116.

6. Miettinen M, Lasota J. Gastrointestinal stromal tumors--definition, clinical, histological, immunohistochemical, and molecular genetic features and differential diagnosis. Virchows Arch 2001; 438: 1-12.

7. Lehnert T. Gastrointestinal sarcoma (GIST)--a review of surgical management. Ann Chir Gynaecol 1998; 87: 297-305.

8. Levine MS, Buck JL, Pantongrag-Brown L, et al. Leiomyosarcoma of the esophagus: radiographic findings in 10 patients. AJR Am J Roentgenol 1996; 167: 27-32.

9. Shojaku H, Futatsuya R, Seto H, et al. Malignant gastrointestinal stromal tumor of the small intestine: radiologic-pathologic correlation. Radiat Med 1997; 15: 189-192.

10. Buckley JA, Fishman EK. CT evaluation of small bowel neoplasms: spectrum of disease. Radiographics 1998; 18: 379-392.

11. Lin YM, Chiu NC, Li AF, et al. Unusual gastric tumors and tumor-like lesions: Radiological with pathological correlation and literature review. World J Gastroenterol 2017; 23: 2493-2504.

12. Bagnolo F, Bonasi U, Scelsi R, Testoni PA. Gastric stromal tumour: a rare neoplasm presenting with gastrointestinal bleeding. Eur J Gastroenterol Hepatol 1998; 10: 791-794.

13. Ghanem N, Altehoefer C, Furtwängler A, et al. Computed tomography in gastrointestinal stromal tumors. Eur Radiol 2003; 13: 1669-78.

14. Sigmund G, Buitrago-Téllez CH, Torhorst J, Steinbrich W. Radiology of gastrointestinal stromal tumor (GÍST) and one new case of Carney's syndrome. Fortschr Röntgenstr 2000; 172: 287-294.

15. He MY, Zhang R, Peng Z, et al. Differentiation between gastrointestinal schwannomas and gastrointestinal stromal tumors by computed tomography. Oncol Lett 2017; 13: 3746-3752.

16. Pannu HK, Hruban RH, Fishman EK. CT of gastric leiomyosarcoma: patterns of involvement. AJR 1999; 173: 369-373.

17. Megibow AJ, Balthazar EJ, Hulnick DH, Naidich DP, Bosniak MA. CT evaluation of gastrointestinal leiomyomas and leiomyosarcomas. AJR 1985; 144: 727-731.

18. Laurent F, Raynaud M, Biset JM, et al. Diagnostic and categorization of small bowel neoplasms: role of computer tomography. Gastrointest Radiol 1991; 16: 115-119. 